\title{
COVID-19 and graft-versus-host disease: a tale of two diseases (and why age matters)
}

\author{
George F. Murphy ${ }^{1}$
}

Received: 21 September 2020 / Revised: 3 November 2020 / Accepted: 4 November 2020 / Published online: 9 December 2020

(c) The Author(s), under exclusive licence to United States and Canadian Academy of Pathology 2020

\begin{abstract}
Disorders involving injury to tissue stem cells that ensure normal tissue homeostasis and repair have potential to show unusually devastating clinical consequences. Acute graft-versus-host disease (aGVHD) is one condition where relatively few cytotoxic immune cells target skin stem cells to produce significant morbidity and mortality. By analogy, SARS-CoV-2 is a vector that initially homes to pulmonary stem cells that preferentially express the ACE2 receptor, thus potentially incurring similarly robust pathological consequences. In older individuals, stem cell number and/or function become depleted due to pathways independent of disease-related injury to these subpopulations. Accordingly, pathologic targeting of stem cells in conditions like aGVHD and COVID-19 infection where these cells are already deficient due to the aging process may have dire consequences in elderly individuals. A hypothesis is herein advanced that, as with aGVHD, lung stem cell targeting is a potential co-factor in explaining age-related severity of COVID-19 infection.
\end{abstract}

\section{Introduction}

It has recently been emphasized by Mueller et al. that both severity and outcome in coronavirus disease 2019 (COVID19 ) is critically dependent on the age of infected individuals [1]. When age appears to play a fundamental role in the severity of an inflammatory disorder, we often focus on agerelated differences that influence responsible effector immune pathways. Indeed, a plethora of age-related inflammatory factors may conspire to produce more virulent infections in the elderly, including those relevant to differences in the innate and adaptive immune systems, specific inflammatory and cytokine pathways, epigenetic regulators, and biological 'clocks' [1]. In the case of susceptibility to and severity of COVID-19 in the aged population, factors such as immunosenescence, alterations in T-cell diversity, and chronic immune activation ('inflammaging') are also likely to impair SARS-CoV-2 virus clearance, with resultant cytokine dysregulation playing a contributing role in enhancing tissue injury, vascular

George F. Murphy

gmurphy@bwh.harvard.edu

1 Department of Pathology, Brigham and Women's Hospital, 75 Francis Street, Boston, MA 02115, USA thrombosis, and multi-organ failure [1]. While these effector immune pathways address key differences between younger and older individuals that relate to differential impact of COVID-19 infection, there remains an additional, potentially covert component of cytopathic injury that also may play an important role in age-related pathogenic disparities: the effect of preferential targeting of specific subpopulations of tissue stem cells critical to lung function and repair.

"Effector-target cell interaction" is a phrase that originally applied to the interplay between immune effector cells and tissue-based cells that they target for the induction of cytopathology. One of the earliest morphologic descriptions of effector-target cell interaction relates to the phenomenon of 'satellitosis', as coined by Woodruff et al. [2], whereby lymphocytes stimulated to recognize and attack foreign antigens (specifically histocompatibility antigens in the case of acute graft-versus-host disease [aGVHD]) aggregate in direct apposition to a centrally-located, degenerating epithelial cell. Whereas in aGVHD, lymphocytes that form 'satellites' about target cells serve as visible biomarkers for the immune assault, more insidious effector pathways such as those involving viral particles, cytotoxic cytokines, and immune reactants may evade histologic observation, although the consequences of their stealth are all too obvious in the form of the tissue injury that they induce and the extent of related organ impairment that they produce. 
The concept of effector-target cell interaction also raises a fascinating question: do some effector pathways selectively seek out and destroy specific subpopulations of target cells? In aGVHD, this indeed appears to be the case, as evidenced in skin and intestinal lesions where tissue stem cells are primary targets of effector pathways set into motion by histocompatability differences inherent to allogeneic bone marrow/stem cell transplantation.

\section{GVHD: a disease where skin stem cells are targeted and destroyed}

One of the mysteries of cutaneous aGVHD based on findings in diagnostic biopsies has long related to how relatively few effector lymphocytes were capable of producing potentially life-threatening epidermal injury [3]. One intriguing possibility was that the effector limb seemed so potent because it specifically attacked only those cells critical to skin homeostasis. This notion was initially validated in 1985 when Sale et al. [4] made a seminal morphological observation: the earliest, and therefore the presumed primary sites, of human epidermal injury in aGVHD involved cell subpopulations that resided at the very tips of epidermal rete ridges, the undulant downward projections whereby the epidermal layer interlocks with capillary-containing dermal papillae. This descriptive insight was biologically significant because three years earlier, Lavker and Sun [5] had discovered that cells that occupied the niches at rete tips are slow-cycling and relatively undifferentiated, and thus had characteristics of epidermal stem cells. In subsequent studies of animal models of aGVHD, we learned that the bulge region of the hair follicle, also an epithelial stem cell niche, was similarly targeted by lymphocytes in early aGVHD $[6,7]$. Only V $\beta$ T-cell subsets from transplanted donor bone marrow that had become clonally expanded during the alloresponse initially homed to and produced satellitosis in these stem cell-rich regions, further confirming the specificity of the early targeting event [8]. We also learned that such epidermal-target cell interactions in aGVHD induced stem cell death via apoptosis, a seeming paradox because among all basal epidermal cells, stem cells were found to be enriched in pathways designed to resist programmed cell death [9, 10]. However, we subsequently discovered that target cell apoptosis in aGVHD was associated with activation of the p53-73 'suicide pathway' designed to protect long-lived stem cells from persisting after they had sustained damage that put host tissue at risk, including potentially deleterious mutations and functionally corrupting danger signals [11]. Of interest, not only did allostimulated donor $\mathrm{T}$ cells prove capable of targeting epithelial stem cells for apoptotic demise, but also cytokines such as tumor necrosis factor alpha $(\mathrm{TNF} \alpha)$ and interleukin 1 beta
(IL-1 $\beta$ ), shared and collaborated in this capacity [8, 12]. These findings were relevant to "cytokine storm", a descriptor historically first used by Ferrara to describe cytokine dysregulation known to typify early phases of acute aGVHD $[13,14]$. Thus, aGVHD came to be understood as a condition where significant epithelial injury was possible based on relatively few cytotoxic effector cells and their mediators as a consequence of early and specific targeting of cells critical for skin homeostasis and repair: epithelial stem cells.

\section{Stem cells in tissue health and aging}

Targeting and resultant apoptotic depletion of epithelial stem cells in aGVHD may have devastating consequences to epidermal integrity. This is because we now know that stem cells in the skin are critical to epidermal and follicular self-renewal, wound healing, and regenerative responses [15-18]. Their deficiency acutely removes the selfperpetuating reservoir necessary to maintain epidermal integrity for protection against infection and fluid and electrolyte depletion. During the ubiquitous aging process, chronic, non-immune impairment of skin stem cell number and/or function results in epidermal atrophy, potentially impacting on the numerous protective roles of the epidermal layer [19]. While multiple mechanisms for stem cell aging and resultant dysfunction have been advanced [20], Fuchs et al. [21] have recently emphasized follicular stem cells as a model in which transcriptional changes, primarily in extracellular matrix genes accompanied by structural anomalies in the immediate stem cell microenvironment, typify the aging process. Moreover, the epidermal thinning that accompanies the aging skin phenotype (contributing to fragility and loss of function and a durable permeability barrier) has recently been attributed to defective physiologic homeostasis whereby age-related loss of epidermal stem cell capacity to express the hemidesmosome component, collagen XVII (COL17A1), results in increased asymmetrical stem cell divisions and a competitive decline that results in the aging phenotype [22].

If skin stem cells are functionally and/or numerically depleted during aging, how might this impact on a disorder like aGVHD where stem cells are specific targets of a cytotoxic effector immune response? Current data indicate that elderly individuals exhibit more severe clinical grades of aGVHD [23]. In murine models of aGVHD, older transplant recipients are reported to be at greater risk for developing disease, with increased morbidity and mortality as well as worsened pathologic and biochemical indices of disease in older animals [24]. While there are likely a multitude of reasons and co-factors that contribute to these observations, including the fact that the immune pathways 
in the elderly, including suppressive limbs, may be perturbed (including regulatory $\mathrm{T}$ cells that normally suppress immunity), one must also consider the possibility that cytotoxic targeting of a critical stem cell population already compromised by age-related decline is a key factor in explaining enhanced severity of aGVHD in the older population.

\section{Stem cells targeting in COVID-19}

Remarkably, skin and lung cells, as well as the pathologic consequences of certain diseases that injure them, have significant parallels. As in skin, the lung also contains stem cells that occupy discrete microenvironmental niches. Clara cells, also known as club cells, are cuboidal, non-ciliated epithelial cells that populate terminal bronchioles where they are interspersed among ciliated cells critical to maintenance of mucociliary clearance of particulates and infectious agents. As reparative cells for ciliated respiratory epithelium, Clara cells serve as facultative progenitors that are senescent in terms of baseline replication, yet metabolically active and capable of re-entering the cell cycle in response to injury [25]. In addition to its function as a tissue-specific stem cell, the Clara cell also functions to detoxify, regulate inflammation, and maintain mucociliary clearance, and therefore has a role in protecting the more distal airways from cytotoxic influences. Within the more distal alveoli where critical air exchange takes place, the alveolar type 2 (AT2) progenitor cell is also a critical source for lung regeneration, a stabilizing influence for host immune responses, and a producer of surfactant necessary to reduce inter-alveolar surface tension [25, 26]. Upon alveolar injury, as occurs in acute respiratory distress syndrome (ARDS) which is a major complication of COVID19 infection, rounded AT2 cells situated in protected corners of alveolar spaces divide and differentiate to replace damaged flattened alveolar type I (AT1) cells that account for most of the alveolar surface area and that are critical to transcellular gas exchange that is responsible for systemic tissue oxygenation. This stem cell-like function of the AT2 cell has been further validated in rodent models [26]. Thus, as in the skin, the lung is endowed with specialized subpopulations of stem cells that are critical to tissue homeostasis and repair. And also like skin stem cells, lung stem cells also exhibit functional decline with age. Recent evidence in murine models has revealed reduced rates of Clara cell self-renewal and differentiation and declining rates of transdifferentiation of AT2 into AT1 cells in association with aging [27]. These events are accompanied by alterations in multiple age-related gene pathways, including those involving proliferation and differentiation, as well as in IGF and TGF $\beta$ signaling. Analogous to skin, this age-related loss of lung stem cell regenerative capacity may have serious implications for both structure and function. Accordingly, it is little wonder that aging is associated with diminished thresholds for acquisition of chronic respiratory disease in humans [28]. As in the case of aGVHD, any disorder with the capability to target lung stem cells and thus compound the pathologic consequences of their agerelated functional compromise over time, would also be anticipated to have the most profound impact in older individuals.

In COVID-19, SARS-CoV-2 may be detected and studied pathologically in the lungs of infected patients, and both bronchial and alveolar epithelial cells have been shown to be infected [29]. The entry point for SARS-CoV-2 into a cell is the host angiotensin-converting enzyme 2 (ACE2) that serves as a receptor for the spike (S) protein of the virus $[30,31]$. Until now, the tissue distribution of ACE2 was poorly understood. However, Ziegler et al. [32] have recently examined this critical issue in humans and animal models using single cell RNA-sequencing (scRNA-seq) and found that specific cell subsets are preferentially involved in $S$ protein/ACE2 interaction. In the lung, a key target for SARS-CoV-2 $S$ protein turns out to be the AT2 stem cell [32]. Notably, this finding is further supported by previous work on SARS-CoV showing differential susceptibility of pulmonary stem/progenitor (Clara) cells expressing the stem cell transcription factor, Oct4, for targeted infection [33]. Thus, current and evolving data implicate early targeting of lung stem cells in COVID-19 infection, the implications of which are potentially profound for understanding some as yet cryptic aspects of disease occurrence and progression in the elderly.

\section{A unified hypothesis: aging, stem cell depletion, and COVID-19}

In both aGVHD and COVID-19, critical epithelial stem cell populations are preferentially targeted in early phases of disease, in one instance by cytotoxic immune pathways, in the other by a viral protein-receptor interaction. Moreover, both conditions are mediated, in part, by cytokines, with 'cytokine storm' playing an important role further driving and amplifying disease pathogenesis. Based on the established critical nature of stem cells to tissue health and to subsequent repair upon injury, as well as the known numerical and/or functional compromise in these subpopulations during the aging process, one may make the following assumption: the effect of COVID-19 on elderly patients, although undoubtedly multifactorial [1], may be in part the result of selective, disease-related depletion/dysfunction of lung stem cells in individuals who already have little to no reserve to fend off further insult to this critical 


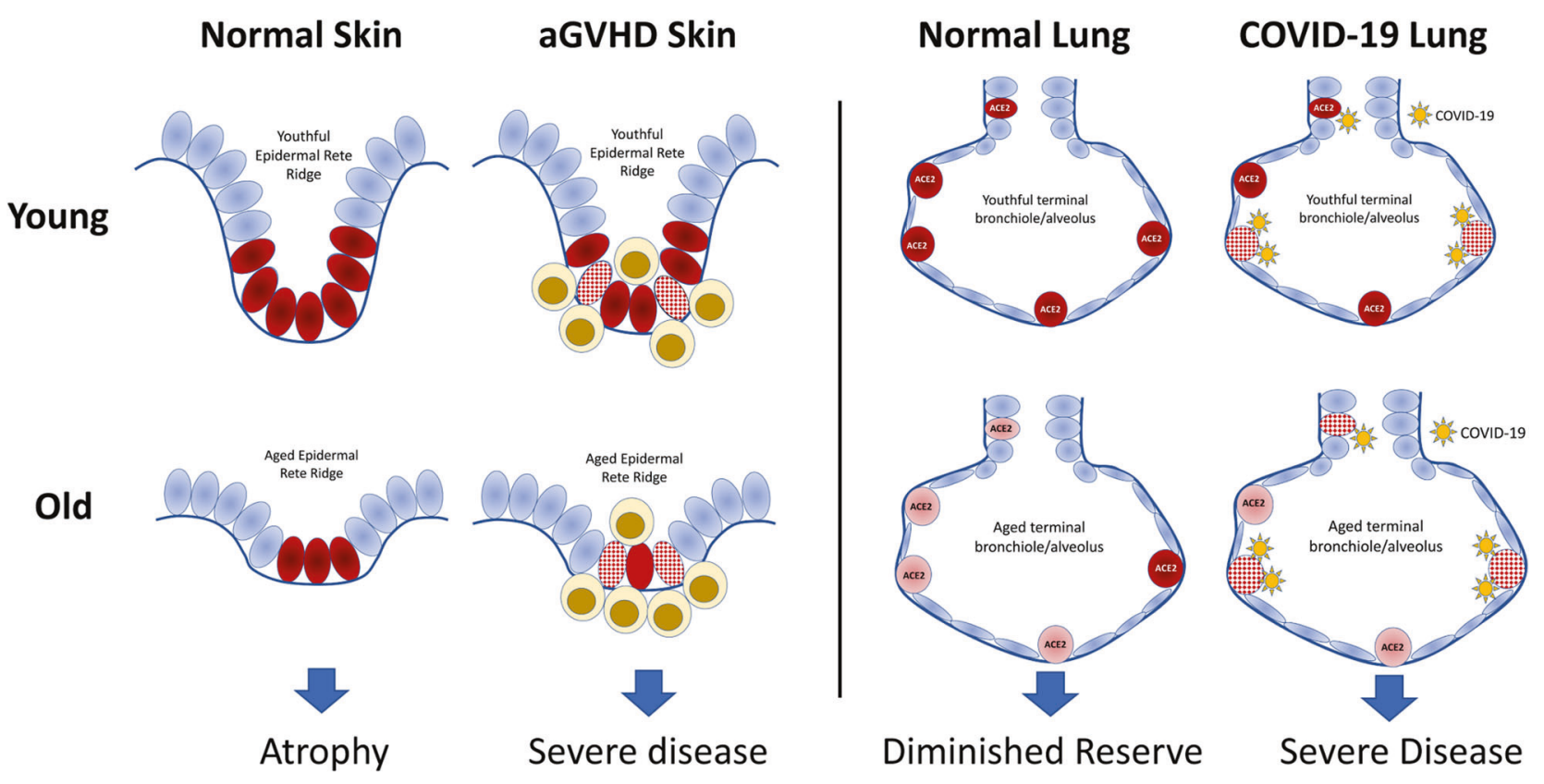

Fig. 1 Schematic representation of stem cell targeting in young and old skin and lung in aGVHD and COVID-19, respectively. In both aGVHD (left) and COVID-19 infection (right), stem cells in affected

tissue are targeted and injured, although they are already compromised in skin and lung from older individuals (also see text).

subpopulation. Alternatively, one could speculate that because older individuals have fewer lung stem cells, the ACE2 receptor may not be as plentiful or prominently displayed for infection to be initiated and propagated as robustly as in younger patients. However, data suggests that in the lung, the primary effect of aging is not actual depletion of stem cells known to express ACE2, but rather their functional decline in terms of proliferative and regenerative potential in response to injury [27].

Figure 1 correlatively illustrates this hypothesis that unifies the common features of cell-specific cytotoxicity in aGVHD in the skin and COVID-19 infection in the lung. Normal skin from a young individual contains well-formed epidermal rete ridges where ovoid basal cells aligned along the undulant basement membrane that separates the epidermal layer from the underlying vascularized dermis. The stem cell component (dark crimson) concentrates at the niche that occupies tips of the rete ridges, as well as the bulge regions of hair follicles (not depicted). Skin from old patients contains fewer functional stem cells needed for epidermal homeostasis, and accordingly the rete ridges are less prominent to absent, resulting in epidermal thinning (atrophy). In addition, hair follicle bulge regions are less robust, often associated with hair thinning or loss. In aGVHD, alloreactive cytotoxic T cells (yellow) and related cytokines (cytokine storm) initially home to and attack stem cells within the rete ridge niches (and bulge regions), resulting in stem cell apoptosis (checkerboard pattern). In contrast to young skin, old skin that contains fewer stem cells necessary for recovery and repair is potentially more vulnerable to this stem cell-directed cytotoxic attack. In normal lung from a young individual, stem cells are interposed among non-stem epithelial lining cells situated along the basement membranes of distal bronchioles and within alveolar spaces into which bronchioles communicate. In older patients, these cells are believed to show decreased functional capacity for homeostasis and repair (pink). Lung stem cells (Clara cells in distal bronchioles, AT2 cells in alveoli) preferentially express the ACE2 receptor for SARSCoV-2, and upon infection, virions (yellow) bind via spike protein to ACE2 that is differentially expressed by stem cells. Intracellular viral replication and associated cytopathic effects [33] culminate in stem cell-directed injury (checkerboard pattern). As with aGVHD, this injury is accentuated in older lungs where stem cell functional capacity is already compromised by the aging process. Associated cytokine storm, as originally described in aGVHD [13, 14], further amplifies the extent of tissue injury. The result is depletion of cells critical for mediation of immune responses and repair, with heightened impact in individuals where these cells are physiologically deficient due to chronologic aging.

\section{Lung stem cells and chronicity of injury due to COVID-19}

Early targeting of critical populations of epithelial stem cells necessary for tissue maintenance and repair in both aGVHD and COVID-19 infection renders the entire epithelial barrier 
more vulnerable to the ravages of the subsequent inflammatory assault that ensues. Patients with aGVHD may progress to more chronic fibrosing manifestations of disease, and the possibility that COVID-19 infection leads to chronic respiratory compromise is now receiving considerable attention [34]. One of the consequences of COVID-19 infection, particularly in the elderly, is ARDS, an established cause of lung fibrosis [35]. Of potential relevance to lung stem cell targeting, aging and COVID-19 infection, recent data suggests that alterations in AT2 cell homeostasis are implicated in the induction of pulmonary fibrosis [36]. Moreover, a rescue therapeutic maneuver of introduction of AT2 cells into bleomycin-damaged fibrotic lungs may actually halt or reverse fibrosis [37], again suggesting a potentially important role for stem cells in the induction and reversal of fibrosing sequellae. Finally, some have averred that anomalies in AT2 cells are actually the primary drivers of pulmonary fibrosis, adding that "aging is the final factor that adds to the pathogenic changes in AT2 cell" [38]. Accordingly, the hypothesis advanced herein is consistent not only with the profound effect that SARS-CoV-2 targeting of lung stem cells incurs on the aging patient population in whom these critically important lung stem cells are already functionally compromised, but also with the notion that lung stem cell targeting in COVID19 infection explains many of the clinical observations of acute disease as well as relates to more chronic consequences.

\section{The next steps: protecting stem cells from COVID-19 pathogenic targeting}

The notion that aGVHD-like epithelial stem cell targeting in COVID-19 infection plays a key role in explaining agerelated morbidity and mortality not only has implications relating to certain aspects of disease pathogenesis, but ironically also may implicate other populations of stem cells as agents of therapy for this potentially deadly infection [39]. In aGVHD, mesenchymal stromal/stem cells (MSCs) that possess immunosuppressive capacities have been successfully deployed [40, 41] to ameliorate disease. Moreover, MSCs are also potentially safe and effective agents for the treatment of acute respiratory distress syndrome (ARDS), a known source of significant morbidity and mortality due to COVID-19 infection [42, 43]. In aGVHD, stem cell apoptosis is in part mediated by TNF $\alpha$-induced $\mathrm{p} 73$ activation which is abrogated in animals genetically deficient in p73 [11], suggesting that stem cell protection may be possible by blocking targeting interactions. Accordingly, decoy strategies to distract COVID-19 spike protein binding away from ACE2 selectively expressed by lung stem cells, particularly if accomplished early in disease, may play similar role in suppressing target cell injury. Such molecular approaches have recently been proposed using soluble recombinant ACE2 [44] or ACE-2 expressing extracellular vesicles [45].

Going forward, it is important that novel therapies that may now emerge consider lung stem cells as potential early targets in early COVID-19 infection, their potential role in mediating age-related disease severity, and the consequences of their cytotoxic targeting in determining the efficiency of regenerative healing during recovery.

Acknowledgements Dr. David Sinclair provided valuable feedback and encouragement.

\section{Compliance with ethical standards}

Conflict of interest The author declares that he has no conflict of interest.

Publisher's note Springer Nature remains neutral with regard to jurisdictional claims in published maps and institutional affiliations.

\section{References}

1. Mueller AL, McNamara MS, Sinclair DA. Why does COVID-19 disproportionately affect older people? Aging. 2020;12:9959-81.

2. Woodruff JM, Butcher WI, Hellerstein LJ. Early secondary disease in the Rhesus monkey. II. Electron microscopy of changes in mucous membranes and expernal epithelia as demonstrated in the tongue and lip. Lab Invest. 1972;27:85-98.

3. Murphy GF, Saavedra AP, Mihm MC. Acute graft-versus-host disease. In: Inflammatory disorder of the skin (atlas of nontumor pathology). Silver Spring, MD: American Registry of Pathology; 2012, pp. 190-5.

4. Sale GE, Shulman HM, Gallucci BB, Thomas ED. Young rete ridge keratinocytes are preferred targets in cutaneous graft-vs-host disease. Am J Pathol. 1985;118:278-87.

5. Lavker RM, Sun TT. Heterogeneity in epidermal basal keratinocytes: morphological and functional correlations. Science. 1982;215:1239-41.

6. Murphy GF, Lavker RM, Whitaker D, Korngold R. Cytotoxic folliculitis in GvHD: evidence of follicular stem cell injury and recovery. J Cutan Pathol. 1991;18:309-14.

7. Cotsarelis G, Sun TT, Lavker RM. Label-retaining cells reside in the bulge area of pilosebaceous unit: Implications for follicular stem cells, hair cycle, and skin carcinogenesis. Cell. 1990;61:1329-37.

8. Whitaker-Menezes D, Jones SC, Friedman TM, Korngold R, Murphy GF. An epithelial target site in experimental graft-versushost disease and cytokine-mediated cytotoxicity is defined by cytokeratin 15 expression. Biol Blood Marrow Trans. 2003;9:559-70.

9. Gilliam AC, Whitaker-Menezes D, Korngold R, Murphy GF. Apoptosis is the predominant form of epithelial target cell injury in acute experimental graft-versus-host disease. J Invest Dermatol. 1996;107:377-83.

10. Zhan Q, Signoretti S, Whitaker-Menezes D, Friedman TM, Korngold R, Murphy GF. Cytokeratin 15-positive basal epithelial cells targeted in graft-versus-host disease express a constitutive anti-apoptotic phenotype. J Invest Dermatol. 2007;127:106-15. 
11. Zhan Q, Korngold R, Lezcano C, McKeon F, Murphy GF. Graftversus-host disease-related cytokine-driven apoptosis depends on p73 in cytokeratin 15-positive target cells. Biol Blood Marrow Trans. 2012;18:841-51.

12. Schmaltz C, Alpdogan O, Murigian SJ, Kappel BJ, Rotolo JA, Ricchetti ET, et al. Donor T-derived TNF is required for graftversus-host disease and graft-versus-tumor activity after bone marrow transplantation. Blood. 2003;101:2440-5.

13. Ferrara JL, Abhyankar S, Gilliland DG. Cytokine storm of graftversus-host disease: a critical effector role for interleukin-1. Trans Proc. 1993;25:1216-7.

14. Ferrara JLM. Cytokine dysregulation as a mechanism of graft versus host disease. Curr Opin Immunol. 1993;5:794-9.

15. Messa KR, Kawaguchi K, Cockburn K, Gonzalez D, Boucher J, Xin $\mathrm{T}$, et al. Homeostatic epidermal stem cell self-renewal is driven by local differentiation. Cell Stem Cell. 2018;23:677-89.

16. Ito M, Liu Y, Yang Z, Nguyen J, Liang F, Morris RJ, et al. Stem cells in the hair follicle bulge contribute to wound repair but not homeostasis of the epidermis. Nat Med. 2005;11:1351-4.

17. Haensel D, Jin S, Sun P, Cinco R, Dragan M, Nguyen Q, et al. Defining epidermal basal cell states during skin homeostasis and wound healing using single-cell transcriptomics. Cell Reports. 2020;30:3932-47.

18. Gonzales KAU, Fuchs E. Skin and its regenerative powers: an alliance between stem cells and their niche. Dev Cell. 2017;43:387-401.

19. Russell-Goldman E, Murphy GF. The pathobiology of skin aging: new insights into an old dilemma. Am $J$ Pathol. 2020;190:1356-69.

20. Ahmed ASI, Sheng MHC, Wasnik S, Baylink DJ, Lau, HW K-. Effect of aging on stem cells. World J Exp Med. 2017;7:1-10.

21. Ge Y, Miao Y, Gur-Cohen S, Gomez N, Yang H, Nikolova M, et al. The aging skin microenvironment dictates stem cell behavior. PNAS. 2020;117:5339-50.

22. Liu N, Matsummura H, Kato T, Ichinose S, Takada A, Namiki T, et al. Stem cell competition orchestrates skin homeostasis and ageing. Nature. 2019;568:344-50.

23. Nakane T, Kanda J, Eto T, Nakamae H, Mori T, Nagamura-Inoue $\mathrm{T}$, et al. Age influences post-GVHD non-relapse mortality in adults with acute GVHD of varying severity following allogeneic hematopoietic cell transplantation. Leukemia Lymphoma. 2015;56:1-29.

24. Ferrara JLM. Pathogenic mechanisms of acute graft versus host disease. Rev Bras Hematol Hemoter. 2002;24:228-34.

25. Reynolds SD, Malkinson AM. Clara cell: progenitor for the bronchiolar epithelium. Int J Biochem Cell Biol. 2010;42:1-4.

26. Olajuyin AM, Zhang X, Ji H-J. Alveolar type 2 progenitor cells for lung injury repair. Cell Death Discov. 2019;5:63.

27. Watson JK, Sanders P, Dunmore R, Rosignoli G, Jule Y, Rawlins EL, et al. Distal lung epithelial progenitor cell function declines with age. Sci Rep. 2020;10:10490.

28. Navarro S, Driscoll B. Regeneration of the aging lung: a minireview. Gerontology. 2017;63:270-80.

29. Schaefer I-M, Padera RF, Solomon IH, Kanjilal S, Hammer MM, Hornick JL, et al. In situ detection of SARS-CoV-2 in lungs and airways of patients with COVID-19. Mod Pathol. 2020; https:// doi.org/10.1038/s41379-020-0595-z.
30. Hoffmann M, Kleine-Weber H, Schroeder S, Kruger N, Herrier T, Erichsen S, et al. SARS-CoV-2 cell entry depends on ACE2 and TMPRSS2 and is blocked by a clinically-proven protease inhibitor. Cell. 2020; https://doi.org/10.1016/j.cell.2020. 02.052 .

31. Wrapp D, Wang N, Corbett KS, Goldsmith JA, Hsieh CL, Abiona O, et al. Cryo-EM structure of the 2019-nCoV spike in the perfusion confirmation. Science. 2020; https://doi.org/10.1126/ science.abb2507.

32. Ziegler CGK, Allon SJ, Nyquist SK, Mbano IM, Miao VN, Tzouanas CN, et al. SARS-CoV-2 receptor ACE2 is an interferonstimulated gene in human airway epithelial cells and is detected in specific cell subsets across tissues. Cell. 2020; https://doi.org/10. 1016/j.cell.2020.04.035.

33. Ling T-Y, Kuo M-D, Li C-L, Yu AL, Huang Y-H, Wu T-J, et al. Identification of pulmonary Oct-4+ stem/progenitor cells and demonstration of their susceptibility to SARS coronavirus (SARS$\mathrm{CoV}$ ) infection in vitro. PNAS. 2006;103:9530-5.

34. Rovere Querini P, De Lorenzo R, Conte C, Brioni E, Lanzani C, Yacoub MR, et al. Post-COVID follow-up clinic: depicting chronicity of a new disease. Acta Biomed. 2020;91:22-28.

35. Spagnolo P, Balestro E, Aliberti S, Cocconcelli E, Biondini D, Casa GD, et al. Pulmonary fibrosis secondary to COVID-19: a call to arms? Lancet Respir Med. 2020;8:750-2.

36. Kobayashi Y, Tata A, Konkimalla A, Katsura H, Lee RF, Ou J, et al. Persistence of a regeneration-associated, transitional alveolar epithelial cell state in pulmonary fibrosis. Nat Cell Biol. 2020;22:934-46.

37. Alvarez-Palomo B, Sanchez-Lopez LI, Moodley Y, Edel MJ, Serrano-Mollar A. Induced pluripotent stem cell-derived lung alveolar epithelial type II cells reduce damage in bleomycininduced lung fibrosis. Stem Cell Res Ther. 2020;11:213.

38. Parimon T, Yao C, Stripp BR, Noble PW, Chen P. Alveolar epithelial type II cells as drivers of lung fibrosis in idiopathic pulmonary fibrosis. Int J Mol Sci. 2020;21:2269.

39. Yu F, Jia R, Wei B. SARS-CoV-2 infection and stem cells: interaction and intervention. Stem Cell Res. 2020; https://doi.org/ 10.1016/j.scr.2020.101859.

40. Dunavin N, Dias A, Li M, McGuirk J. Mesenchymal stromal cells: what is the mechanism of acute graft-versus-host disease. Biomedicines. 2017;5:39.

41. Amorin B, Alegretti AP, Valim V, Pezzi A, Laureano AM, da Silva MAL, et al. Mesenchymal stem cell therapy and acute graftversus-host disease: a review. Hum Cell. 2014;27:137-50.

42. Horie S, Gonzalez HE, Laffey JG, Masterson CH. Cell therapy in acute respiratory distress syndrome. $\mathrm{J}$ Thorac Dis. 2018;10:5607-20.

43. Leng Z, Zhu R, Hou W, Feng Y, Yang Y, Han Q, et al. Transplantation of ACE2(-) mesenchymal stem cells improves the outcome of patients with COVID-19 pneumonia. Aging Dis. 2020;11:216-28.

44. Batlle D, Wysocki J, Satchell K. Soluble angiotensin-converting enzyme 2: a potential approach for coronavirus infection therapy? Clin Sci. 2020;134:543-5.

45. Inal JM. Decoy ACE2-expressing extracellular vesicles that competitively bind SARS-CoV-2 as a possible COVID-19 therapy. Clin Sci. 2020;134:1301-4. 